\title{
Identification of PCR-based markers linked to wood splitting in Eucalyptus grandis
}

\author{
Eugenia Barros ${ }^{\mathrm{a}^{*}}$, Steve Verryn ${ }^{\mathrm{b}}$ and Marianne Hettasch ${ }^{\mathrm{b}}$ \\ ${ }^{a}$ CSIR-Bio/Chemtek, P.O.Box 395, Pretoria 0001, South Africa \\ ${ }^{\mathrm{b}}$ CSIR-Environmentek, P.O.Box 395, Pretoria 0001, South Africa
}

(Received 5 July 2001; accepted 17 April 2002)

\begin{abstract}
Wood splitting is a defect in eucalyptus which results in considerable losses when converting logs to solid wood products. Commonly in forestry, molecular markers are identified through studying pedigrees from a single cross. This limits the application of these markers to that population. Here we report the identification of putative molecular markers linked to wood splitting in an open-pollinated Eucalyptus grandis population. Although the power to detect molecular markers in this population is low, the resultant markers are likely to be more robust and be transferred to non-related populations. Bulked segregant analysis was used in the identification of markers from high and low splitting individuals that were selected by means of backward selection using Best Linear Prediction. The bulks were screened for differences using amplified fragment length polymorphic and random amplified polymorphic DNA primers. Following regression analysis one putative sequence characterized amplified region has been linked to splitting.
\end{abstract}

wood splitting / molecular markers / Eucalyptus grandis / BLP

Résumé - Identification de marqueurs PCR liés au défaut de fente du bois chez Eucalyptus grandis. La fente du bois est un défaut chez l'eucalyptus qui entraîne des pertes considérables lors de la transformation du bois en produits finis massifs. Classiquement chez les arbres forestiers, les marqueurs moléculaires sont identifiés en étudiant les descendants d'un seul croisement. Ceci limite l'application de ces marqueurs à cette seule population. Dans cette étude, nous avons cherché à identifier des marqueurs moléculaires liés vraisemblalement au défaut de fente du bois dans une population d'Eucalyptus grandis issue de pollinisation libre. Bien que le pouvoir de détection de marqueurs moléculaires dans cette population soit faible, il est probable que les marqueurs résultants soient plus robustes et transférables à des populations non apparentées. Une analyse de ségrégation en mélange (BSA) a été utilisée pour l'identification de marqueurs à partir d'individus contrastés pour le défaut de fente et choisis par sélection à rebours à l'aide de la BLP (Best Linear Prediction). Les deux lots ainsi constitués ont été examinés pour leur différence à l'aide de marqueurs ADN (AFLP et RAPD). À la suite d'une étude de régression, nous avons pu relier une SCAR putative au défaut de fente du bois.

défaut de fente du bois / marqueurs moléculaires / Eucalyptus grandis / BLP

\section{INTRODUCTION}

Growing pressure to stop the harvesting of indigenous forests has resulted in an international shift towards plantation grown hardwoods. To date only approximately $10 \%$ of the world's needs for hardwoods come from plantations. Eucalyptus is an important plantation species and is the most widely planted hardwood species in the tropical and subtropical regions.
Eucalyptus grandis is the most extensively grown eucalypt species in South Africa. This species boasts good growth in most forestry areas. It is suitable for pulp wood production but its suitability for sawn timber production is limited by its tendency to split. Wood splitting results in significant losses when converting logs into solid wood products. Currently wood splitting can only be assessed after felling the trees from the age of 6 years onwards due to late expression of the trait. The need for destructive sampling and

\footnotetext{
* Correspondence and reprints

Tel.: +2712 841 3221; fax: +2712841 3651; e-mail: ebarros@ csir.co.za
} 
the late expression of the trait are serious constraints in breeding programmes for sawn timber. Wood splitting is a heritable trait but is also under environmental influence. Narrow sense heritablities of between 0.3 and 0.6 (Verryn, 2001, personal communication) have been reported. It is assumed that wood splitting is a polygenic trait.

At present conventional tree breeding techniques have been successfully used to improve the quality of eucalypts plantations to produce high quality logs. However, the availability of molecular markers for wood splitting would be of great advantage for any Eucalyptus sawn timber breeding programme.

In forestry, marker development is generally based on a limited number of crosses with the resultant markers being only applied to a small number of trees. Due to the limited application and the high costs involved, marker-assisted selection is not yet routinely applied in tree breeding programmes [9]. In our research we explored the possibility of developing more robust markers that could also be used on non-related E. grandis populations.

The aim of our research was to use the tools provided by molecular biology in combination with quantitative genetics and statistics to develop a non-destructive and early screening method to identify $E$. grandis seedlings or young plants that are low splitters.

\section{MATERIALS AND METHODS}

Two unrelated, open pollinated E. grandis trials were used in this study. The first trial, called the development trial was used to identify putative molecular markers linked to splitting. This trial is an open-pollinated population grown from local unimproved $\mathrm{P}_{0}$ selections from South African plantations. A total of 10 high splitting and 16 low splitting trees were selected from this trial by means of backward selection. Backward selection ranks individuals on their progenies' performance. The backward selection was performed by means of Best Linear Prediction (BLP). Theoretically the BLP gives the best correlation between the true genetic value and the predictive genetic value. This was done using the programme Matgen 5.6, a BLP package for unbalanced index selection in tree breeding [5].

The second trial was used as a verification trial and the individuals were selected by means of forward selection where individuals are evaluated based on their own performance. A total of 21 high splitting and 31 low splitting trees were selected from a total of 750 trees. The verification trial is an open-pollinated population that has been established from seed imported from Florida, USA.

The trees were evaluated for splitting using a splitting score which took into consideration the number, the length and width of the cracks. The splitting scores were then corrected for the diameter of the tree.

DNA was isolated from leaf material collected from each of the 78 trees belonging to the 2 trials following the modified protocol of Rogers and Bendich [3]. Two molecular marker techniques were used in the generation of DNA fingerprints for the identification of polymorphisms. These were RAPDs (Random Amplified Polymorphic DNA) [8] and AFLPs (Amplified Fragment Length Polymorphism) [6]. All the resulting bands were scored on a scale ranging from 0 to 4 according to the intensity of the bands. A score of 0 represented the absence of a band and a score of 4 a very dark band.

In addition the bulked segregant analysis (BSA) [2, 7] technique was used to identify markers linked to the gene/s coding for splitting/non-splitting. This technique has proved to be very successful in populations resulting from a single cross that segregate for the trait of interest. We used the BSA technique on an open-pollinated population, which is likely to be in linkage equilibrium. Linkage disequilibrium between marker alleles and QTL alleles, is however, a prerequisite for marker detection, as unlinked markers and markers in linkage equilibrium with QTL are expected to be randomly distributed across bulks [1]. Only markers that are very tightly linked to the QTL are likely to show linkage disequilibrium. The power of detection is drastically reduced by targeting only very closely linked markers. High numbers of potential markers need to be analysed. If, however, markers are found, these are likely to be more robust and may be valid for more than just the population that was used to develop them. Another challenge is to find more than one marker linked to splitting since we assume this trait to be polygenic.

Two DNA bulks/pools were initially made for each trial, one being the high splitting bulk and the other the low splitting bulk. The principle of DNA pooling/bulking is the grouping together of informative individuals for a specific trait so that a particular genomic region can be studied in a randomised genetic background of unlinked loci [7]. The RAPD technique uses random 10-base oligonucleotides as primer and the polymerase chain reaction (PCR) to amplify specific DNA fragments. The RAPD primers were obtained from UBC RAPD primer set. The RAPD primers that showed polymorphisms between the bulks were then tested in the individuals making the bulks. The polymorphic bands were considered "putative markers" if they were present in 4 or more individuals of one of the unbulked populations and absent in the other unbulked population. In total 828 RAPD primers were tested in the first trial.

We attempted to convert the "putative" RAPD markers into SCAR (sequence characterized amplified regions) markers. The SCARs were derived by cloning and sequencing the 2 ends of the amplified products of the RAPD marker. The sequence was then used to design oligonucleotide primer pairs of 19-24mer that result in the reproducible amplification of single loci when high annealing temperatures are used. The AFLP technique uses genomic DNA digested with restriction enzymes as a PCR template. In this technique the binding between short PCR primers and DNA restriction fragments is very specific. We used the Mse 1/EcoR1 and Mlul/Msel restriction enzyme systems. The fingerprints generated were scored for polymorphisms in the same way as the RAPD fingerprints.

The statistical analysis aimed at finding models that use the scores of a limited number of fragments to predict wood splitting in E. grandis. Using the data sets generated from the RAPD scores for the development trial, a number of models were developed to predict splitting using the statistical package SAS [4]. The data set which was used for the development of the models consisted of over a thousand fragments for each of the 26 trees of the first trial - this corresponds to data generated from 91 RAPDs and 4 AFLPs. Stepwise (forward) regression was performed on the data set to identify any set of fragments that were possibly linked to either high or low splitting. The fragments that together described the variation in the splitting index value were combined in a regression equation that may be used to predict splitting.

Regression models were generated using the model development trial data set and were tested on the verification trial data set. The models that were developed were verified by entering the scores of the fragments from the verification data set into the regression equations. This resulted in the predicted values for wood splitting for the 


\section{$\lambda$ H1 H2 H3 H4 H5 H6 H7 H8 H9 H10 HB LB L1 L2 L3 L4 L5 L6 L7 L8 L9 L10 L11 L12 L13 L14 L15 L16 FH FL $\lambda$}

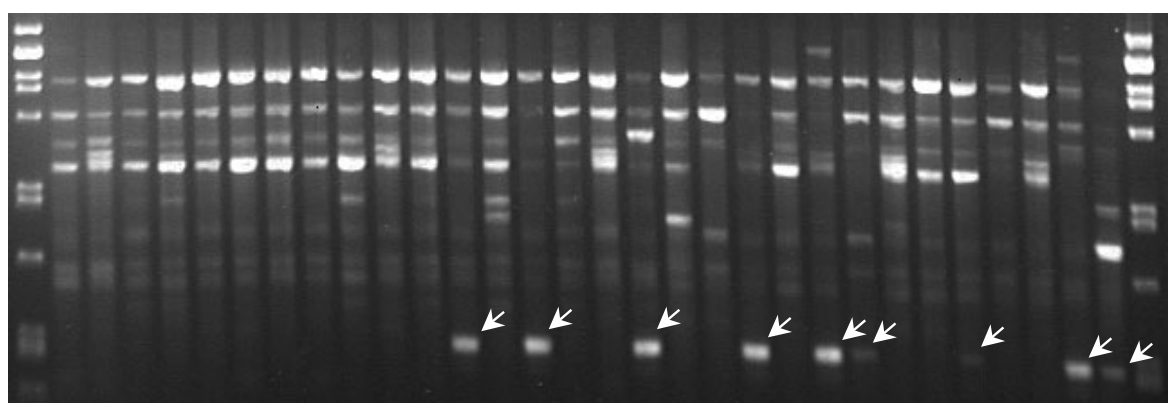

Figure 1. Amplification products using RAPD A primer to identify markers linked to splitting. The polymorphism linked to splitting is marked by arrows. First lane is $\lambda$ PstI marker and the other lanes correspond to the 26 development trial individuals. $\mathrm{H}$ means high splitters and L means low splitters.

31 low and 21 high split trees. The predicted value was then compared to the observed value by means of a correlation coefficient. Significant and high correlations may indicate linkage between the DNA bands and wood splitting genes.

\section{RESULTS}

A total of 118 RAPD primers showed polymorphisms in the first trial and were used to generate fingerprints of the 52 unbulked individuals that formed the verification trial. Figure 1 shows the RAPD fingerprint of the unbulked individuals of the development trial for RAPD A. Two RAPD polymorphic bands were converted into SCARs. The SCAR markers were verified by hybridisation with the corresponding DIG-labelled RAPD bands. The results of the hybridisation showed that RAPD B putative marker was successfully converted into a SCAR marker and was present in the individuals for which the RAPD "putative marker" was originally present (figure 2). The RAPD A putative marker did not hybridise to the SCAR marker suggesting that the band cloned was not the correct band (results not shown). Two AFLP markers have also been converted into SCAR markers and the data is being analysed statistically.
Many regression models have been tested. The best correlation that was obtained was around 0.4 (significant at the 5\% level). Some of the models gave no correlation between the predicted and the observed splitting scores, whereas some gave relatively weak but significant correlations. A perfect match between the observed and the expected value would give a correlation coefficient of 1.0. More models will be tested to further improve the correlation. The correlation of the predicted versus obtained splitting scores from the best model is shown in figure 3.

\section{DISCUSSION}

Wood splitting seems to be a polygenic trait and it is therefore highly unlikely that a single DNA marker will be sufficient to distinguish between high and low splitting trees. The procedure of forward regression allows the development of models that are based on several DNA fragments. This increases the chances of finding fragments that are linked to more than one gene. A large number of fragments have to be screened and a large number of models have to be tested to increase the chances of obtaining a useful model. Once a useful
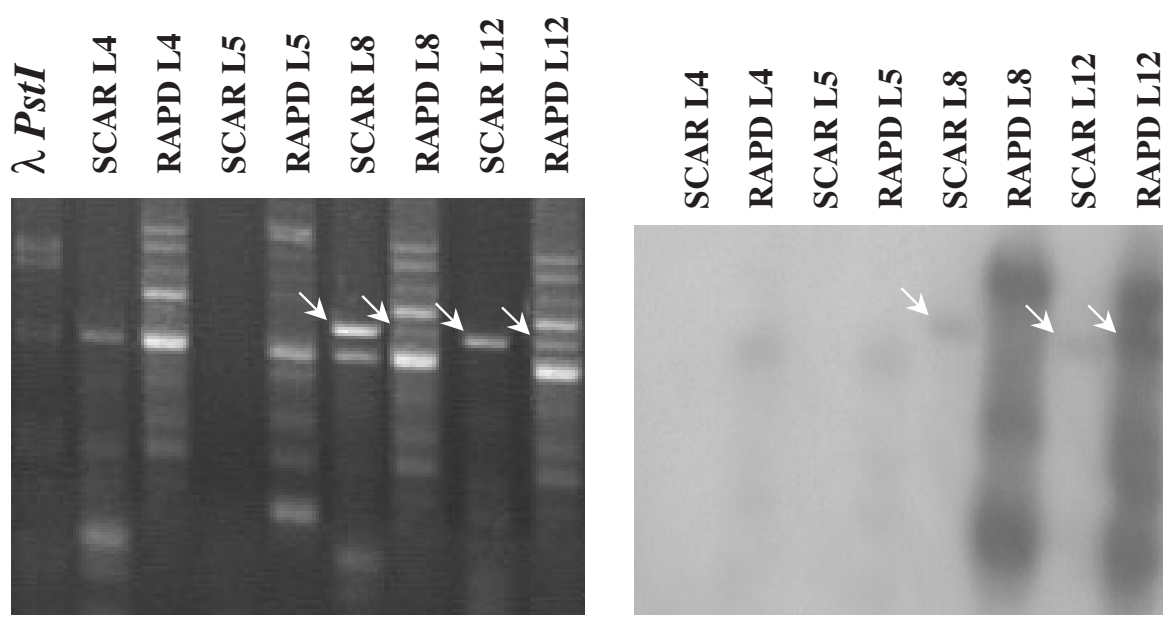

Figure 2. Hybridization of the cloned RAPD putative marker $B$ (arrowed) to a Southern Blot of 4 low splitting individuals (L4, L5, L8 and L12). First lane is $\lambda$ PstI marker. 


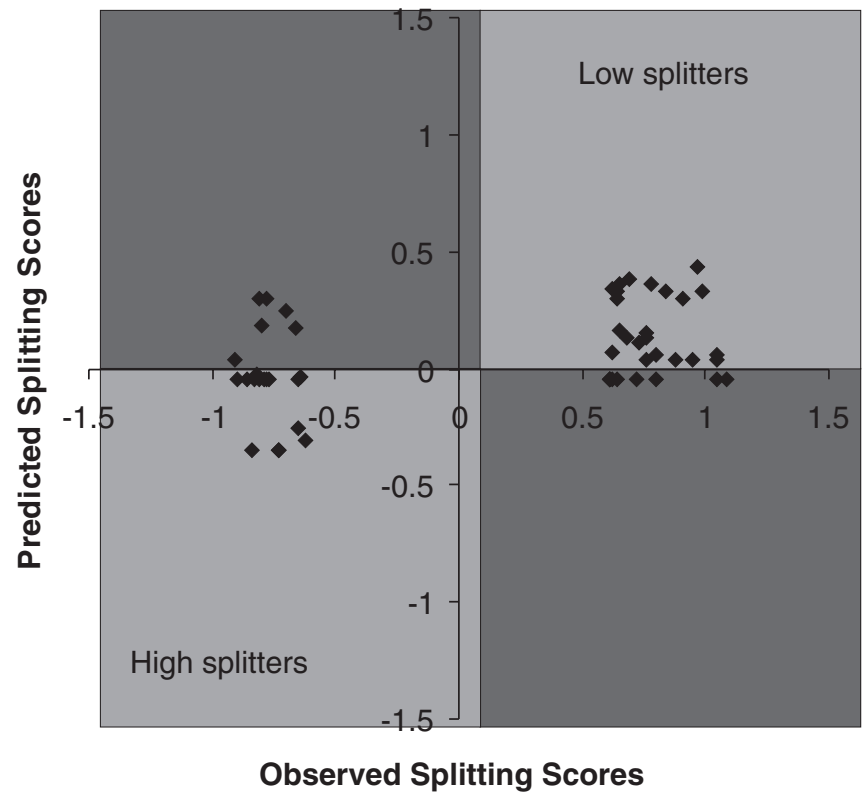

Figure 3. Scores predicted by a model versus observed splitting scores. The trees falling into the first and third quadrants have phenotypes which were predicted incorrectly.

model is obtained with a reasonable correlation a splitting index will be generated that will be used in breeding programmes. However the fact that the 2 trials are unrelated it may to a certain extent explain our difficulty in obtaining markers linked to splitting. Although ultimately we aimed at identifying a wood splitting marker that would work in basically all $E$. grandis material and would be linked to all the genes that are involved in splitting this may be too ambitious. The approach taken in this study using random populations and BSA had a low power to detect marker-QTL association compared to the traditional approaches which uses controlled-cross mapping populations. However the marker gen- eration in the traditional approach is limited to the mapping population from which it was generated.

We have identified 2 other trials that are derived from the development trial and will be used as verification trials. A splitting index will then be generated for the development trial to be used by breeders using progeny derived from this original trial. We also need to identify a trial derived from the verification trial that can be used to verify the markers identified for this trial. Similarly a splitting index will be generated to be used by breeders using progeny originated from this trial. We are very confident that we will succeed in generating a set of molecular markers that can be used to help eucalyptus breeders to screen their tree material for low splitters.

\section{REFERENCES}

[1] Lynch M., Walsh B., Genetics and analysis of quantitative traits, Sinauer Associates, Inc., Sunderland, USA, 1998.

[2] Michelmore R.W., Paran I., Kesseli R.V., Identification of markers linked to disease resistance genes by bulked segregant analysis: A rapid method to detect markers in specific genomic regions using segregating populations, Proc. Natl. Acad. Sci. USA 88 (1991) 9828-9832.

[3] Rogers O.S., Bendich A.J., Extraction of DNA from plant tissues, in: Gene Research Manual, 1986, pp. 1-6.

[4] SAS Institute Inc., SAS Users Guide: Basics (1985) 5th Edition. Cary, NC, USA.

[5] Verryn S.D., Roux C.Z., A memory-friendly algorithmic strategy for Best Linear Prediction (BLP), in: Tree Improvement for Sustainable Tropical Forestry-Conference Proceeding, Dieters M.J., Matheson A.C., Nikles D.G., Harwood C.E., Walker S.M. (Eds.), Caloundra, Australia: Queensland Forestry Research Institute, 1996.

[6] Vos P., Hogers R., Bleeker M., Reijans M., van de Lee T., Hornes M., Frijters A., Pot J., Peleman J., Kuiper M., AFLP: a new technique for DNA fingerprinting, Nucleic Acids Res. 23 (1995) 4407-4414.

[7] Wang G.L., Paterson A.H., Assessment of DNA pooling strategies for mapping of QTLs, Theor. Appl. Genet. 88 (1994) 355-361.

[8] Williams J., Kubelik A., Livak K., Rafalski J., Tingey S., DNA polymorphisms amplified by arbitrary primers are useful as genetic markers, Nucleic Acids Res. 18 (1990) 6531-6535.

[9] Yanchuk A.D., The role and implications of biotechnological tools in forestry, Unasylva. 52 (2001) 53-61.

To access this journal online:

www.edpsciences.org 